\title{
Dispersive-Dissipative Eddy Parameterization in a Barotropic Model
}

\author{
BALASUbramanya T. NADigA \\ Earth and Environmental Sciences, Los Alamos National Laboratory, ${ }^{*}$ Los Alamos, New Mexico \\ Len G. MARgOlin \\ Center for Nonlinear Studies, Los Alamos National Laboratory, Los Alamos, New Mexico
}

16 May 2000 and 13 March 2001

\section{ABSTRACT}

\begin{abstract}
Recently a new class of coarse-grained equations, known as $\alpha$ models, have been proposed for the mean motion of an ideal incompressible fluid. The use of one such model to represent the time-mean component of a turbulent $\beta$-plane circulation characterized by potential vorticity mixing is considered. In particular, the focus is on the wind-driven circulation in a shallow ocean basin, a problem well studied as a prototype of more realistic ocean dynamics. The authors demonstrate the ability of an $\alpha$ model to reproduce qualitatively the structure of a four-gyre circulation that forms (in the time mean) when the barotropic vorticity equation is driven by a symmetric, double-gyre wind forcing, and when the dissipation is weak. This is offered as a first step in assessing the utility of the $\alpha$-model approach to simulating more complex geophysical flows.
\end{abstract}

\section{Introduction}

In a variety of turbulent geophysical flows, the tendency of potential vorticity to become homogenized inside a closed streamline has been recognized (Rhines and Young 1982), and this homogenization has served as a basis for eddy parameterizations. In most such parameterizations, the divergent part of the eddy potential vorticity flux is modeled as a downgradient transfer, resulting in a purely dissipative closure. In this paper we consider an alternate route to homogenization of potential vorticity and present preliminary results from our investigation of a class of fluid descriptions to model geophysical fluid turbulence.

The evolution of the time-mean flow in the simple case of the barotropic vorticity equation is given by

$$
\frac{\partial \bar{q}}{\partial t}+\boldsymbol{\nabla} \cdot(\overline{\mathbf{u}} \bar{q})+\boldsymbol{\nabla} \cdot \overline{\mathbf{u}^{\prime} q^{\prime}}=\bar{F}+\bar{D}
$$

where $q=\nabla^{2} \psi+\beta y$ is the potential vorticity, $\psi$ is the

\footnotetext{
* Los Alamos National Laboratory is operated by the University of California.

Corresponding author address: Dr. Balasubramanya T. Nadiga, Earth and Environmental Sciences, MS-B296, Los Alamos National Laboratory, Los Alamos, NM 87545.

E-mail: balu@lanl.gov
}

velocity streamfunction, $F$ is the forcing, $D$ is the dissipation, an overbar denotes the time mean, and a prime denotes the difference from the mean (see below for other notation). In (1) it is clearly only the divergent component of the eddy flux of potential vorticity $\overline{\mathbf{u}^{\prime} q^{\prime}}$ that can drive the mean flow, and the aforementioned downgradient closure for (1) takes the form

$$
\left(\overline{\mathbf{u}^{\prime} q^{\prime}}\right)_{\mathrm{div}}=-\kappa \nabla \bar{q},
$$

where $\left(\overline{\mathbf{u}^{\prime} q^{\prime}}\right)_{\text {div }}$ denotes the divergent component of $\overline{\mathbf{u}^{\prime} q^{\prime}}$. This closure is purely dissipative.

Holm et al. (1998) proposed a model for the mean motion of ideal incompressible fluids wherein the nonlinear fluid transport term $\mathbf{u} \cdot \nabla \mathbf{u}$ is modified into the advection of a momentum density $\mathbf{v}=\left(1-\alpha^{2} \nabla^{2}\right) \mathbf{u}$ by the usual velocity $\mathbf{u}$ plus other nonlinear dispersive terms:

$$
\frac{\partial \mathbf{v}}{\partial t}+\mathbf{u} \cdot \boldsymbol{\nabla} \mathbf{v}-\alpha^{2}(\boldsymbol{\nabla} \mathbf{u})^{\mathrm{T}} \cdot \nabla^{2} \mathbf{u}=-\boldsymbol{\nabla} p, \quad \boldsymbol{\nabla} \cdot \mathbf{u}=0 .
$$

(Note that, when $\alpha$ is set to zero, the usual Euler equations result.) In a complementary fashion, the transport velocity $\mathbf{u}$ for such a flow may be viewed as Helmholtzsmoothed relative to the advected quantity $\mathbf{v}$. Such a model is obtained by introducing a length scale $\alpha-$ determined by problem dynamics-in the Euler-Poincare formalism and conserving an energy 


$$
\frac{1}{2} \int\left(|\mathbf{u}|^{2}+\alpha^{2}|\nabla \mathbf{u}|^{2}\right) d \mathbf{x}
$$

that also accounts for the spatial fluctuations of the velocity field in place of the usual kinetic energy

$$
\frac{1}{2} \int|\mathbf{u}|^{2} d \mathbf{x}
$$

Conservation of the latter kinetic energy results in the usual Euler equations. For details of the derivation of (3) and its other interesting properties, the reader is referred to Holm et al. (1998) and Shkoller (1998).

Using a vorticity streamfunction formulation in two dimensions, (3) can be written as

$$
\frac{\partial \zeta}{\partial t}+\left(1-\alpha^{2} \nabla^{2}\right)^{-1} J\left[\psi,\left(1-\alpha^{2} \nabla^{2}\right) \zeta\right]=0,
$$

where $\zeta=\mathbf{k} \cdot \nabla \times \mathbf{u}=\nabla^{2} \psi$ is the (relative) vorticity. When $\alpha=0$ in (6), the two-dimensional (inviscid and unforced) Euler equation

$$
\frac{\partial \zeta}{\partial t}+J[\psi, \zeta]=0
$$

is obtained.

Until recently, most work on equations of the type of (3) seems to have been of a mathematical nature. It is only recently, with the rederivation by Holm et al. (1998), that their potential as models for coarse-grained hydrodynamics has been recognized. Chen et al. (1998) used a steady and parallel flow solution of a viscous $\alpha$ model to fit turbulent channel data, and Chen et al. (1999) considered a viscous $\alpha$ model to study homogeneous and isotropic turbulent scalings in three dimensions. Nadiga (2000) considered statistical equilibria of (6) and showed that the scaling of the equilibrium states of the model for conserved energy and enstrophy reproduced the corresponding scaling for the Euler equations on the large scales, while greatly deemphasizing the importance of the small scales. Nadiga and Shkoller (2001), in the content of two-dimentional turbulence, showed how scales much larger than $\alpha$ can be affected due to an enhancement of the inverse cascade of energy by $\alpha$.

In this paper, we examine the utility of an extension of (6) to the $\beta$-plane barotropic vorticity equation by modeling a turbulent flow that can be directly realized in eddy-permitting simulations of the barotropic vorticity equation. The outline of the rest of the paper is as follows: In the next section, we present the $\alpha$ model corresponding to the barotropic vorticity equation. In section 3, we briefly recapitulate the phenomenon of the formation of a time-mean four-gyre circulation in a strongly turbulent $\beta$-plane barotropic vorticity equation flow driven by the classic double-gyre wind forcing. In section 4 we show that, with a judicious choice of boundary conditions, the $\alpha$-barotropic vorticity equation can model the important, eddy-driven components of the time-mean four-gyre circulation. We summarize our results in section 5 .

\section{The barotropic vorticity equation and its $\alpha$ model}

The unforced and inviscid barotropic vorticity equation may be written as

$$
\frac{\partial q}{\partial t}+J[\psi, q]=0,
$$

where $q=\operatorname{Ro} \zeta+y$ is the potential vorticity, $\zeta=\nabla^{2} \psi$ is the relative vorticity, $\psi$ is the velocity streamfunction,

$$
J[\psi, q]=-\frac{\partial \psi}{\partial y} \frac{\partial q}{\partial x}+\frac{\partial \psi}{\partial x} \frac{\partial \psi}{\partial x},
$$

and Ro is the Rossby number. We consider a rectangular, closed basin on a beta plane with the $y$ coordinate $(-L$ $\leq y \leq L)$ increasing northward and with the $x$ coordinate $(0 \leq x \leq L)$ increasing eastward. We define the Rossby number by

$$
\operatorname{Ro}=\left(\frac{\delta_{i}}{L}\right)^{2}=\frac{U}{\beta L^{2}},
$$

where $\delta_{i}$ is the inertial boundary layer thickness, the reference length $L$ is the width of the domain, $U$ is a reference velocity, and $\beta$ is the (north-south) gradient of rotation. For future reference, we mention that in the forced-dissipative cases to be considered, we will choose the reference velocity to be the Sverdrup velocity corresponding to the applied wind stress forcing. With such a choice, the magnitude of the wind stress curl forcing will be unity.

Holm et al. (1998) construct an $\alpha$ model for the EulerBoussinesq equations in the presence of rotation (assuming a $\beta$-plane approximation) and stratification and refer to them as the $\alpha$-Euler-Boussinesq equations [their (10)]. While the the reader is referred to Holm et al. (1998) for details of that construction, we consider the specialization of the so-called $\alpha$-Euler-Boussinesq equations to two dimensions. On taking the curl of the $\alpha$-Euler-Boussinesq equations [(10) of Holm et al. 1998] in two dimensions, we obtain

$$
\frac{\partial q}{\partial t}+\left(1-\alpha^{2} \nabla^{2}\right)^{-1} J\left[\psi,\left(1-\alpha^{2} \nabla^{2}\right) q\right]=0 .
$$

We call this the $\alpha$-barotropic vorticity equation. When $\alpha$ is set to zero in the above equation, the usual unforced and inviscid barotropic vorticity equation (8) is obtained.

In (9), $\alpha$ is a nondimensional turbulence correlation length, which we consider to be the ratio of a length scale appropriate to the eddy activity to be modeled and the reference length $L$. We remark that (9) may also be written in the form 

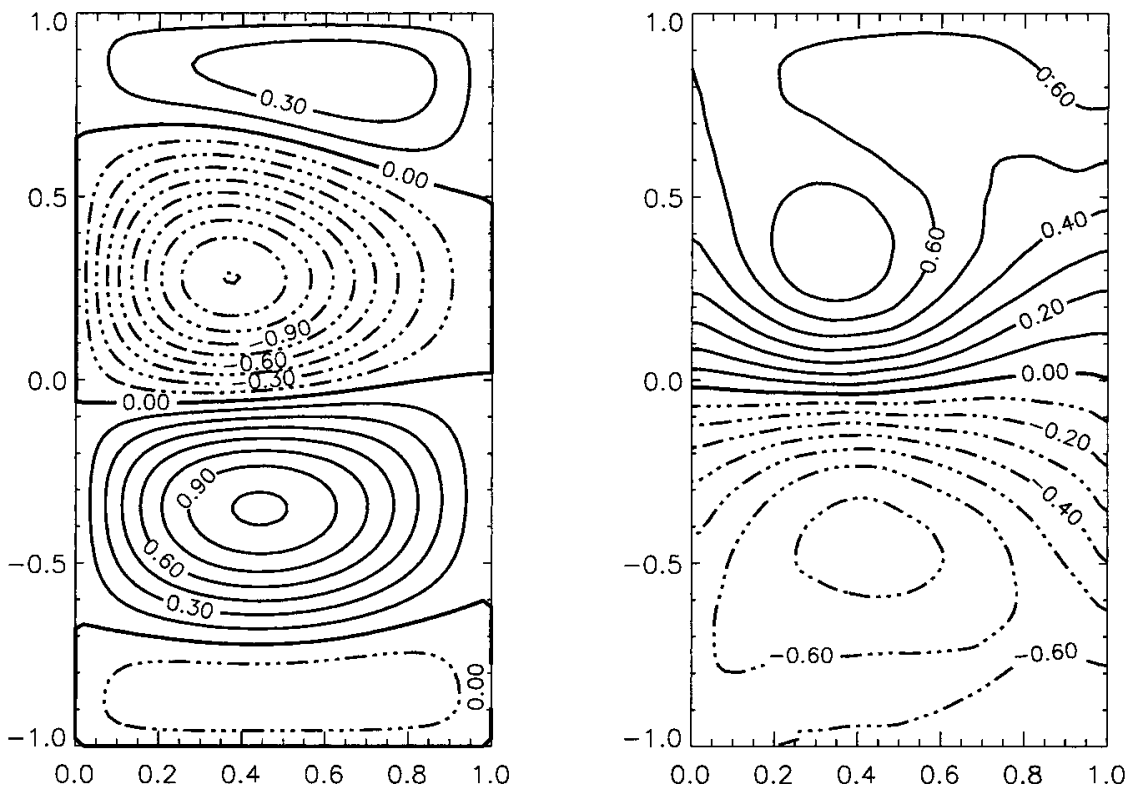

FIG. 1. Contour plots of the time-averaged streamfunction and potential vorticity. The Rossby number for this double-gyre wind-forced beta-plane circulation is 0.01 , the Munk scale is 0.04 with superslip sidewall boundary conditions, and the grid spacing is 0.02 . The contour intervals are 0.15 for streamfunction and 0.10 for potential vorticity. The instantaneous flow is highly turbulent.

$$
\frac{\partial q^{\alpha}}{\partial t}+J\left[\psi, q^{\alpha}\right]=0,
$$

where

$$
q^{\alpha}=\left(1-\alpha^{2} \nabla^{2}\right) q .
$$

This form makes transparent the conservative and advective nature of the model equation. In this preliminary study, in writing (11), we have considered $\alpha$ to be constant. However, in a more realistic situation $\alpha$ would be variable and could, for example, depend on the local value of eddy kinetic energy.

Even though the net number of derivatives in (9) is the same as in (8), in the sense that the Helmholtz operator acting on $q$ is reversed by an inverse Helmholtz operation after the Jacobian is evaluated in (9), it remains that a new boundary condition has to be specified for $q$. This is evident by considering the evolution of $q^{\alpha}$ as given, for example, by (10) and (11). To the extent that the outer gyres are driven by the (eddy) mixing of potential vorticity (Greatbatch and Nadiga 2000, hereafter GN), we choose for this new boundary condition

$$
\frac{\partial q}{\partial n}=0 .
$$

\section{Four-gyre circulation with double-gyre wind forcing}

When the barotropic vorticity equation (8) is forced by the Ekman pumping resulting from a double-gyre wind stress $[F=\sin (\pi y)]$ and the dissipation $D$ is weak, the instantaneous flow is highly turbulent, but the timemean flow shows a four-gyre structure (see GN). Figure 1 shows the time-mean streamfunction and potential vorticity contours for such a case where the Rossby number was 0.01 , and where a Laplacian mixing of momentum

$$
D=\left(\frac{\delta_{2}}{L}\right)^{3} \nabla^{2} \zeta,
$$

with an associated Munk scale $\left(\delta_{2} / L\right)$ of 0.04 was used in conjunction with superslip boundary conditions. The flow was resolved on a $51 \times 101$ grid giving a grid spacing $\Delta x / L$ of 0.02 , enough to marginally resolve the Munk scale. Unless stated otherwise, all computations presented in this article were carried out using secondorder finite difference spatial discretization with Arakawa Jacobian and a (nominally) fifth-order, adaptive time step, embedded Runge-Kutta Cash-Karp algorithm time marching scheme. (For details, see GN.)

In Fig. 1, the two inner gyres circulate in the same direction as conventional wind-driven gyres, while the two outer gyres at the northern and southern ends of the basin circulate in the opposite direction and are driven by the eddy flux of potential vorticity (as can be seen by the mean vorticity balance for each of the four gyres in Table 1). Furthermore, it is clear from Table 1 that the dominant balance is between the wind forcing and the (divergence of the) eddy flux of potential vorticity (with explicit dissipation playing a minor role). When 
TABLE 1 . The mean vorticity balance for the eddy-permitting run. The dominant balance is between forcing and the divergence of eddy flux of potential vorticity.

\begin{tabular}{lrrr}
\hline \hline \multicolumn{1}{c}{ Gyre } & $-\overline{J\left(\psi^{\prime}, q^{\prime}\right)}$ & \multicolumn{1}{c}{$\bar{F}$} & \multicolumn{1}{c}{$\bar{D}$} \\
\hline Southern outer & 0.148 & -0.141 & -0.008 \\
Subtropical wind-driven & 0.476 & -0.492 & 0.017 \\
Subpolar wind-driven & -0.421 & 0.438 & -0.016 \\
Northern outer & -0.203 & 0.195 & 0.007 \\
\hline
\end{tabular}

the importance of the eddy flux of potential vorticity is reduced by increasing the explicit dissipation, the outer counterrotating gyres disappear and only a conventional double-gyre circulation appears in the time mean (see Fig. 8 of GN). The formation of such a time-mean fourgyre circulation, given a double-gyre wind forcing, is quite robust and does not depend sensitively on either the Rossby number, the form of dissipation, or the boundary conditions as long as the dissipation is weak.

While it is clear that the parameter regime we consider must not be terribly geophysically relevant because a time-mean, four-gyre circulation is not observed in nature, we note that a choice of a larger value of dissipation that would have resulted in a turbulent double-gyre circulation would have worked fine, but for the disadvantage of rendering more intricate the process of evaluating parameterizations.

Following the tendency exhibited in Fig. 1 for potential vorticity to mix due to eddy activity in the northern and southern parts of the domain, the qualitative aspects of the time-mean flow are obtainable (in a coars- er-resolution run) by a downgradient parameterization of the eddy flux of potential vorticity as in (2) (see GN). Then, the eddy-parameterized model equations are

$$
\frac{\partial q}{\partial t}+J[\psi, q]=F+D, \quad D=\nabla \cdot(\kappa \nabla q),
$$

where the variables are to be interpreted as time averages; Fig. 2 shows the result. In this parameterized case with the same Rossby number as before, a spatially uniform $\kappa$ corresponding to a Munk scale of about 0.17 was used, and the resolution was cut in half in each direction. Balance is, by design, between the forcing and dissipation terms; this is verified in Table 2. We remark that the correspondence between the time mean of the eddy-permitting simulation (Fig. 1) and the steady state of the downgradient parameterized model (Fig. 2) is only qualitative.

\section{An alternative parameterization}

In this section, we consider steady states of the forced-dissipative version of the $\alpha$-barotropic vorticity equation (9),

$$
\frac{\partial q}{\partial t}+\left(1-\alpha^{2} \nabla^{2}\right)^{-1} J\left[\psi,\left(1-\alpha^{2} \nabla^{2}\right) q\right]=F+D,
$$

as a model for the eddy-driven mean flow previously discussed. Recognizing that the problem is forced and the left-hand side of (14) is conservative, one is forced to introduce dissipation $D$ in order to ensure that kinetic
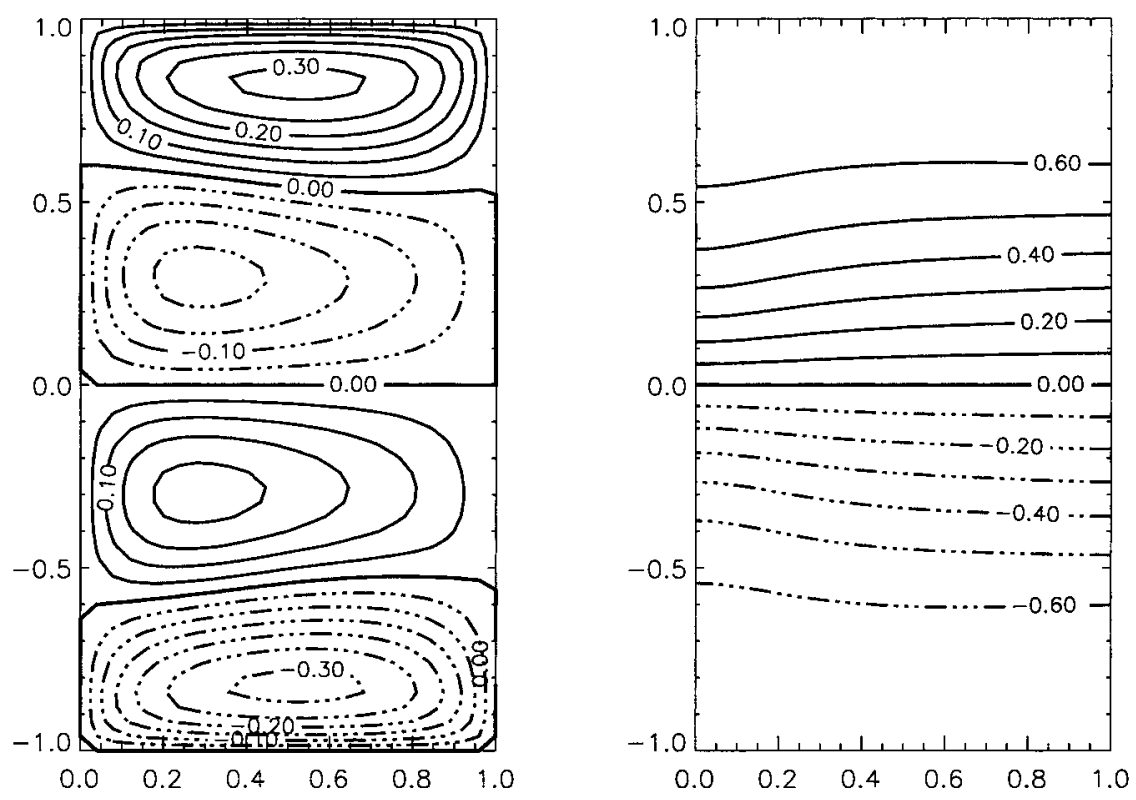

FIG. 2. The steady-state fields using a downgradient parameterization. Rossby number is 0.01 and the dissipative term, a Laplacian mixing of potential vorticity, arises from a parameterization of the divergent component of the eddy potential vorticity flux as a down the gradient of mean potential vorticity. The associated Munk scale is 0.17 and a nonormal potential vorticity flux boundary condition is used. The grid spacing is 0.04 . The contour intervals are 0.05 for streamfunction and 0.10 for potential vorticity. 
TABLE 2. The mean vorticity balance for the downgradient-parameterization case. The balance is by design between the forcing and dissipation.

\begin{tabular}{lrr}
\hline \hline \multicolumn{1}{c}{ Gyre } & \multicolumn{1}{c}{$F$} & \multicolumn{1}{c}{$D$} \\
\hline Southern outer & -0.267 & 0.268 \\
Subtropical wind-driven & -0.369 & 0.367 \\
Subpolar wind-driven & 0.369 & -0.367 \\
Northern outer & 0.266 & -0.268 \\
\hline
\end{tabular}

energy and potential enstrophy remain bounded. In this context, we briefly discussed in the previous section 1) how eddies in the simulation that permitted eddies tended to mix potential vorticity and 2) how such a mixing was achievable in a parameterized run by using a downgradient approximation for the eddy potential vorticity flux, resulting in a dissipation of the form indicated in (13). Now, in the context of the $\alpha$ parameterization contained in the left-hand side of (14), if we were to choose the dissipation $D$ to be of the same form as in (13), our conclusion would not be compelling. That is to say, it would be difficult to separate out the effects of the $\alpha$ parameterization and the dissipation term (related to the downgradient approximation). To this end, we choose a form of dissipation that is not effective in modeling the downgradient nature of the eddy flux of potential vorticity. We choose Rayleigh damping of relative vorticity $\zeta$ :

$$
D=-\frac{\delta_{s}}{L} \zeta
$$

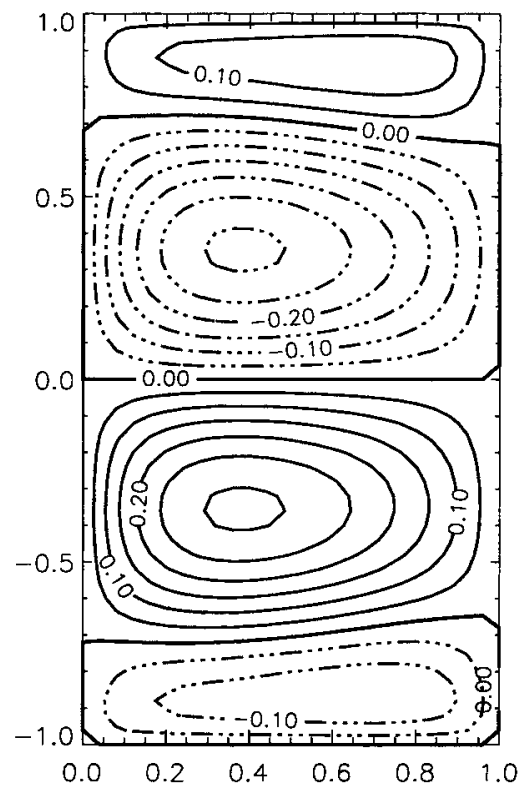

That this form of dissipation acting alone is ineffective in tending to homogenize potential vorticity will be demonstrated later.

In Fig. 1, the return western boundary flow in the conventional gyres extends to about $0.45 \mathrm{~L}$ in the eastwest direction and the outer gyres extend to about $0.40 \mathrm{~L}$ latitudinally. Thus, the scale over which eddy activity affects the mean flow is about $0.45 \mathrm{~L}$, and we choose this as the length scale $\alpha$ in (14). (While we could examine the wavenumber spectra of the eddy-permitting runs to obtain $\alpha$, in this preliminary study we are limited by the small dynamic range of such spectra.) Figure 3 shows the steady-state circulation resulting from the parameterized model. In this parameterized setup, while the domain size is the same as before, the resolution has been cut in half in each direction compared to the eddying run: $\Delta x / L=0.04$. The averaging length scale $\alpha$ is $0.45 L$ and a Rayleigh friction corresponding to a Stommel boundary layer scale of $0.07 \mathrm{~L}$ was used at the same value of Rossby number of 0.01 . While we chose $\alpha$ as roughly the average of the lengths of the two nonlinear features as previously discussed, qualitatively similar results are obtained for quite a wide range of $\alpha$ $(0.3 L \leq \alpha \leq 0.7 L)$. Again, with this alternative parameterization, the four-gyre structure and the homogenization of potential vorticity in the region of the outer gyres is evident and robust.

As further proof that it is indeed the $\alpha$ parameterization that is modeling the effects of eddies and thereby mixing potential vorticity, we repeat the previous run but with

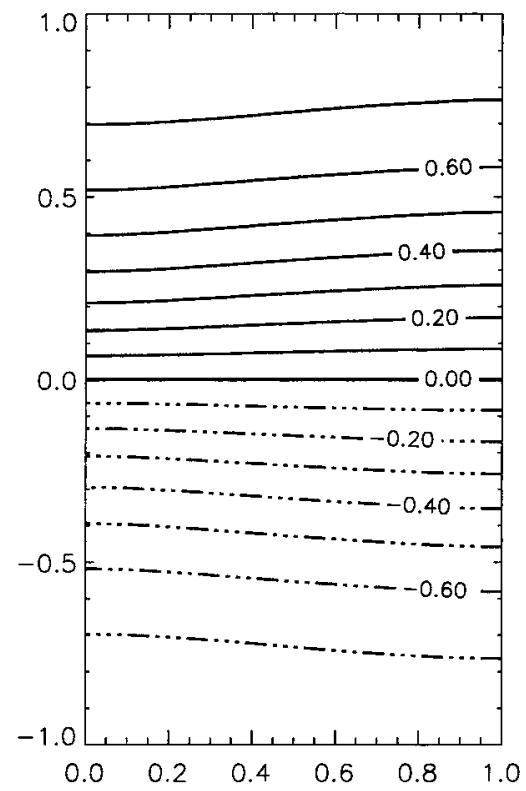

FIG. 3. The steady-state fields from the $\alpha$-parameterized model. Rossby number is 0.01 and the dissipative term is chosen to be Rayleigh damping of relative vorticity to ensure that this term does not represent downgradient transfer. The Stommel scale associated with this dissipation operator is $0.07 \mathrm{~L}$. The grid spacing is 0.04 . The contour intervals are 0.05 for streamfunction and 0.10 for potential vorticity. 

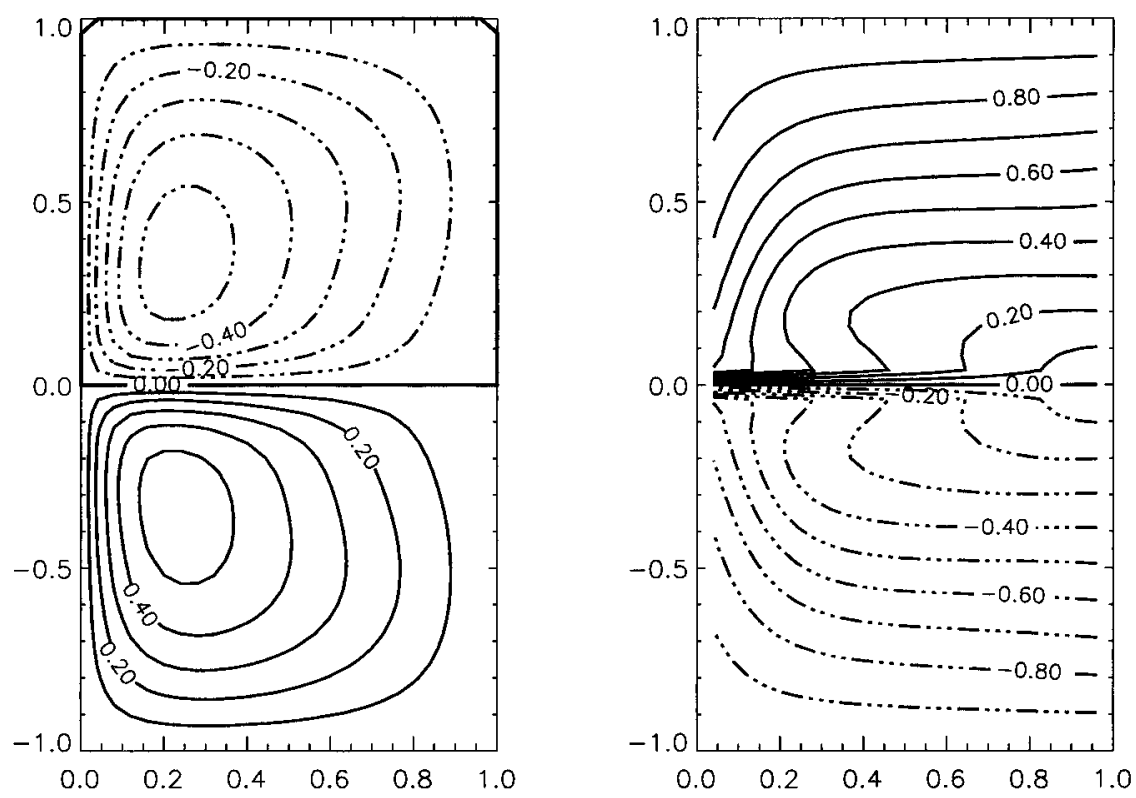

FIG. 4. The steady-state fields from the previous run with $\alpha$ parameterization turned off. The contour intervals are 0.10 for streamfunction and 0.10 for potential vorticity.

$\alpha$ set to zero. The steady-state solution for this case, shown in Fig. 4, corresponds to a steady double gyre. For this case, the Arakawa form of the Jacobian, which conserves both energy and enstrophy, was not used since it leads to grid scale oscillations in the potential vorticity field near the western boundary. Instead, following Veronis (1966), we chose a form of the Jacobian that only conserves energy, but has the useful property that the boundary values of relative vorticity are never needed. It is for this reason that potential vorticity is not defined on the boundary points in Fig. 4.

Indeed, what is interesting with the $\alpha$-parameterized run is that there is no explicit downgradient transfer term and the modeling of eddies results from a modification to the advective term. To the extent that the modeling of eddy effects in the $\alpha$ model is adiabatic, the $\alpha$ model is similar to the Gent-McWilliams parameterization of the effects of eddies in noneddy resolving models (see, e.g., Gent et al. 1995), wherein a bolus or eddy-transport velocity is added to the velocity that advects scalars.

The vorticity balance for the case with dispersive pa-

TABLE 3 . The mean vorticity balance for the dispersive-parameterization case. Note the importance of the inviscid model term in this three-term balance as compared to the forcing-dissipation balance in the downgradient-parameterization case. Here $H$ stands for the Helmholtz operator $\left(1-\alpha^{2} \nabla^{2}\right)$.

\begin{tabular}{lcrr}
\hline \hline \multicolumn{1}{c}{ Gyre } & $H^{-1} J[\psi, H q]$ & \multicolumn{1}{c}{$F$} & \multicolumn{1}{c}{$D$} \\
\hline Southern outer & 0.388 & -0.136 & -0.252 \\
Subtropical wind-driven & 0.264 & -0.500 & 0.235 \\
Subpolar wind-driven & -0.265 & 0.500 & -0.235 \\
Northern outer & -0.387 & 0.135 & 0.252 \\
\hline
\end{tabular}

rameterization is shown in Table 3. If each of the gyres is considered individually, it is clear that equilibrium is achieved by a three-term balance with the inviscid nonlinear dispersive term playing a significant role. This is unlike the forcing-dissipation balance achieved when the downgradient parameterization is used. Further, if one considers the two halves of the basin separated by the zero wind stress curl line, the dominant balance in the case of $\alpha$ parameterization turns out to be between the wind stress curl forcing and the inviscid model term, with the dissipation playing only a minor role in close analogy with the mean vorticity balance of the eddypermitting case.

Finally, we note that it is only the qualitative aspects of the four-gyre structure that are reproduced in the new parameterization considered, in that neither the magnitude of the circulation nor the structure of the potential vorticity field is accurately reproduced. This is 1) indicative of the need for a spatially variable $\alpha$ in the dispersive parameterization, and 2) consistent with a similar lack of detailed correspondence between the steady-state circulation that results when a spatially constant diffusivity is used in the downgradient parameterization case and the time-mean circulation of the eddy-permitting simulation.

\section{Summary and discussion}

These are preliminary results from a study of the applicability of the $\alpha$ models to modeling geophysical fluid turbulence, and more work is necessary to understand the relevance of these models to describing the oceans and the atmosphere. That apart, we have shown that a 
time-mean circulation of the barotropic vorticity equation with an important eddy-driven component can be reproduced using one such eddy parameterization. This parameterization arises from a specialization to the barotropic vorticity equation of a class of ideal incompressible fluid models proposed to model mean flow. These models are derived by considering the mean dispersive effects of rapid fluctuations over a length scale $\alpha$.

From the point of view of geophysical fluid dynamics, this new parameterized model shows that the tendency of eddies to mix potential vorticity in certain situations can be successfully parameterized in a manner very different from the usual dissipative mixing term that arises from assuming a downgradient form for the eddy flux of potential vorticity. As to whether the new model is indeed better than the usual downgradient mixing parameterization is a question that requires further study.

Acknowledgments. The authors gratefully acknowledge discussions with Richard Greatbatch, and thank the referees for their constructive suggestions. Computer resources for this work were made available in part by the Institute for Geophysics and Planetary Physics at Los Alamos National Laboratory. This work was supported by the U.S. Department of Energy's Climate Change Prediction Program (CCPP).
REFERENCES

Chen, S., C. Foias, D. D. Holm, E. Olson, E. S. Titi, and S. Wynne, 1998: The Camassa-Holm equations as a closure model for turbulent channel flow. Phys. Rev. Lett., 81, 5338-5341.

- D. D. Holm, L. Margolin, and R. Zhang, 1999: Direct numerical simulations of the Navier-Stokes alpha model. Physica D, 133, 66-83.

Gent, P. R., J. Willebrand, T. J. McDougall, and J. C. McWilliams, 1995: Parameterizing eddy-induced tracer transports in ocean circulation models. J. Phys. Oceanogr., 25, 463-474.

Greatbatch, R. J., and B. T. Nadiga, 2000: Four-gyre circulation in a barotropic model with double-gyre wind forcing. J. Phys. Oceanogr., 30, 1461-1471.

Holm, D. D., J. E. Marsden, and T. S. Ratiu, 1998: Euler-Poincaré models of ideal fluids with nonlinear dispersion. Phys. Rev. Lett., 80, 4273-4277.

Nadiga, B. T., 2000: Scaling properties of an inviscid mean-motion fluid model. J. Stat. Phys., 98, 935-948.

- S. Shkoller, 2001: Enhancement of the inverse-cascade of energy in the two-dimensional Lagrangian-averaged NavierStokes equations. Phys. Fluids, 13, 1528-1531.

Rhines, P. B., and W. R. Young, 1982: Homogenization of potential vorticity in planetary gyres. J. Fluid Mech., 122, 347-367.

Shkoller, S., 1998: Geometry and curvature of diffeomorphism groups with $\mathrm{H}^{1}$ metric and mean hydrodynamics. J. Funct. Anal., 160, 337-365.

Veronis, G., 1966: Wind-driven ocean circulation-Part 2. Numerical solutions of the non-linear problem. Deep-Sea Res., 13, 13-55. 